\title{
End Stage Renal Disease due to Acute Pyelonephritis: A Case Report
}

\section{Masatomo Kaneko ${ }^{1,2}$, Eiichi Konishi ${ }^{3}$, Toru Matsugasumi ${ }^{4}$, Hidefumi Taniguchi ${ }^{5}$, Akira Onishi ${ }^{2}$, Hideo Seki $^{6}$, Emi Inui $^{2}$, Naoki Imada ${ }^{2}$ and Osamu Ukimura ${ }^{1}$}

${ }^{1}$ Department of Urology, Kyoto Prefectural University of Medicine, Japan

${ }^{2}$ Department of Urology, Nishijin Hospital, Japan

${ }^{3}$ Department of Surgical Pathology, Kyoto Prefectural University of Medicine, Japan

${ }^{4}$ Department of Urology, Kyoto Chubu Medical Center, Japan

${ }^{5}$ Department of Urology, Ayabe City Hospital, Japan

${ }^{6}$ Department of Urology, Tojinkai Hospital, Japan

Received: February 19, 2018; Published: February 22, 2018

*Corresponding author: Masatomo Kaneko, Department of Urology, Kyoto Prefectural University of Medicine, 6028566 Kajii-cho, Kamigyo-ku, Kyoto, Japan, Tel: +81-75-251-5595; Email: mskaneko@koto.kpu-m.ac.jp

\section{Abstract}

An 89-year-old woman was admitted with complaining of fever and anuria. Physical examination revealed pyuria and left costovertebral angle (CVA) tenderness. A CT scan showed atrophy of the right kidney, and perirenal fat stranding in the left abnormally enlarged kidney. Based on a clinical diagnosis of acute kidney injury (AKI) due to acute pyelonephritis (APN) in the left, intravenous antibiotics, fluid, and hemodialysis was started. After healing of APN, she could not wean from hemodialysis. In order to prevent AKI due to APN from progression to end stage renal disease (ESRD), further studies about new treatment are needed.

Keywords: End Stage Renal Disease; Acute Kidney Injury; Acute Pyelonephritis; Solitary Kidney

Abbreviations: ESRD: End Stage Renal Disease; AKI: Acute Kidney Injury; APN: Acute Pyelonephritis; CVA: Costovertebral Angle; KDIGO: the Kidney Disease Improving Global Outcomes Introduction

\section{Introduction}

APN is an inflammation of the kidney and renal pelvis [1]. APN frequently cause mild AKI, but, irreversible renal function loss is rare [2]. It is presumed that solitary kidney, pregnancy, indwelling urinary catheter or use of non-steroidal anti-inflammatory drugs (NSAIDs) is the cause of severe AKI due to APN [3]. We reported a case of 89-year-old woman with solitary functioning kidney who was diagnosed AKI due to APN.

\section{Case Report}

An 89-year-old female was referred to us with complaining of fever and anuria for one day. She had the past history of chronic kidney disease (CKD) stage G4A1, and hypertension. She had not taken NSAIDs. When she was admitted, her temperature was $37.6^{\circ} \mathrm{C}$, blood pressure $131 / 46 \mathrm{mmHg}$, pulse $60 / \mathrm{min}$. On physical examination, she had left CVA tenderness, nausea, vomiting, and disturbance of consciousness. She denied other symptoms. Laboratory analysis showed white blood cells (WBC) $12 \times 10^{3}$ $/ \mu \mathrm{L}$, hemoglobin $8.0 \mathrm{~g} / \mathrm{dL}$, hematocrit $24.0 \%$, platelets $220 \times$ $10^{3} / \mu \mathrm{L}$, neutrophils $88 \%$, blood urea nitrogen (BUN) $85 \mathrm{mg} /$ $\mathrm{dl}$, creatinine $6.75 \mathrm{mg} / \mathrm{dl}$, C-reactive protein (CRP) $20.07 \mathrm{mg} / \mathrm{dl}$, plasma sodium $125 \mathrm{~m} \mathrm{Eq/l}$, potassium $6.2 \mathrm{~m} \mathrm{Eq/l}$, chloride $99 \mathrm{~m}$ $\mathrm{Eq} / \mathrm{l}$. Urinalysis showed urine protein $(2+)$, specific gravity $\geqq 1.030$, red blood cells (RBC) 5-9/high-power field (HPF), WBC $\geqq 100 / H P F$. Immunoglobrins and autoantibodies associated with glomerular nephritis were all normal level.

A non-contrast abdominal CT scan revealed the atrophic right kidney and perirenal fat stranding around left enlarged kidney. Hydronephrosis and urolithiasis was not detected (Figure 1). The diagnosis of AKI stage 3 in the Kidney Disease Improving Global Outcomes (KDIGO) classification due to APN in solitary functioning 
kidney was suggested. Intravenous dose of ceftriaxone $2 \mathrm{~g}$ daily, hydration, furosemide $40 \mathrm{mg}$, calcium gluconate $8.5 \% 10 \mathrm{~mL}$, and 6 units of regular insulin in $50 \mathrm{ml}$ of $50 \%$ dextrose were initiated. In spite of these treatments, anuria was prolonged, her conscious level decreased, and blood chemistry showed creatinine $7.3 \mathrm{mg} / \mathrm{dl}$ and plasma potassium $6.2 \mathrm{mEq} / \mathrm{l}$ on her 2 nd hospital day. Hemodialysis was started. During hospitalization, Escherichia coli were cultured from her urine and blood. According to result of the culture, ceftriaxone was changed to piperacillin/tazobactam 2.25g IV q12hr on the 5th day. Her CRP and WBC were almost normalized on day 20. After recovery from APN, anuria continued, therefore she could not wean from hemodialysis. Because of patient's refusal, renal biopsy was not performed.

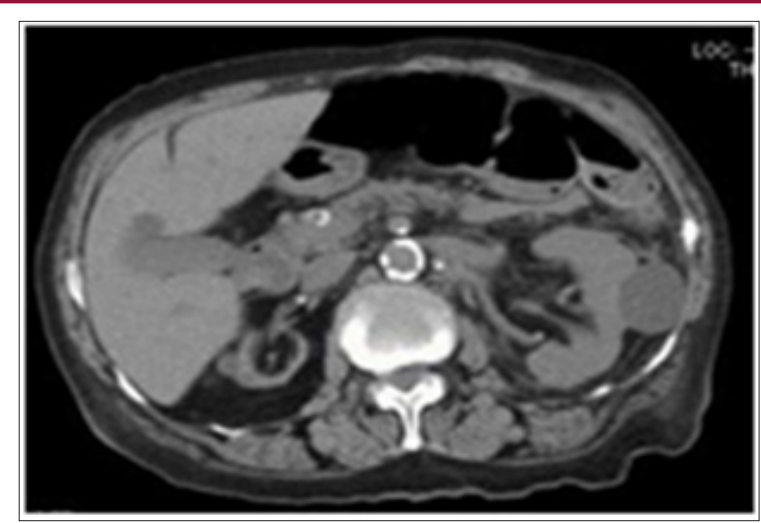

Figure 1: A CT scan revealed the atrophic right kidney, and perirenal fat stranding around the left enlarged kidney.

\section{Discussion}

Recent study revealed AKI in elderly increased the incidence of ESRD, and the risk among patients with both AKI and CKD was higher [4]. It is considered that advanced age, increased Charlson comorbidity index score [5] during AKI episode, hypoalbuminemia, pasthistory of diabetes mellitus, hypertension, heartfailure, and CKD are the risk factor of CKD progression after AKI [6]. The mechanism of AKI to CKD progression has not been clarified. Takaori et al. recently indicated that selective proximal tubule injury can trigger fibrosis, reduced Epo production, glomerulosclerosis, atubular glomeruli, and distal tubule injury by the animal model of CKD [7]. In this case, an elder woman whose medical history included hypertension and CKD with APN in solitary kidney lost renal function irreversibly in spite of prompt treatments. Treatments for preventing AKI to ESRD progression has not been developed, it is presumed to protect proximal tubules and treatments of fibrosis are effective [7].

\section{Conclusion}

In order to prevent acute kidney injury due to acute pyelonephritis from progression to end stage renal disease, further studies about new treatment are needed.

\section{References}

1. Schaeffer AJ, Matulewict RS, Klumpp DJ (2016) Infections of the Urinary Tract. In: Wein AJ, Kavoussi LR, Partin AW (Edn) Campbell-Walsh Urology. $11^{\text {th }}$ edition Elsevier, pp. 237-304.

2. Johnson JR, Russo TA (2018) Acute Pyelonephritis in Adults. N Engl J Med 378(1): 48-59.

3. Turner ME, Weinstein J, Kher K (1996) Acute renal failure secondary to pyelonephritis. Pediatrics 97(5): 742-743.

4. Ishani A, Xue JL, Himmelfarb J, Eggers PW, Kimmel PL, et al. (2009) Acute kidney injury increases risk of ESRD among elderly. J Am Soc Nephrol 20(1): 223-228.

5. Charlson ME, Pompei P, Ales KL, Mackenzie CR (1987) A new method of classifying prognostic comorbidity in longitudinal-studies: development and validation. J Chronic Dis 40(5): 373-383.

6. Goldstein SL, Jaber BL, Faubel S, Chawla LS (2013) AKI transition of care: a potential opportunity to detect and prevent CKD. Clin J Am Soc Nephrol 8(3): 476-483.

7. Takaori K, Nakamura J, Yamamoto S, Nakata H, Sato Y, et al. (2016) Severity and Frequency of Proximal Tubule Injury Determines Renal Prognosis. J Am Soc Nephrol 27(8): 2393-2406.

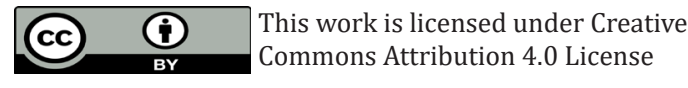

Submission Link: http://biomedres.us/submit-manuscript.php

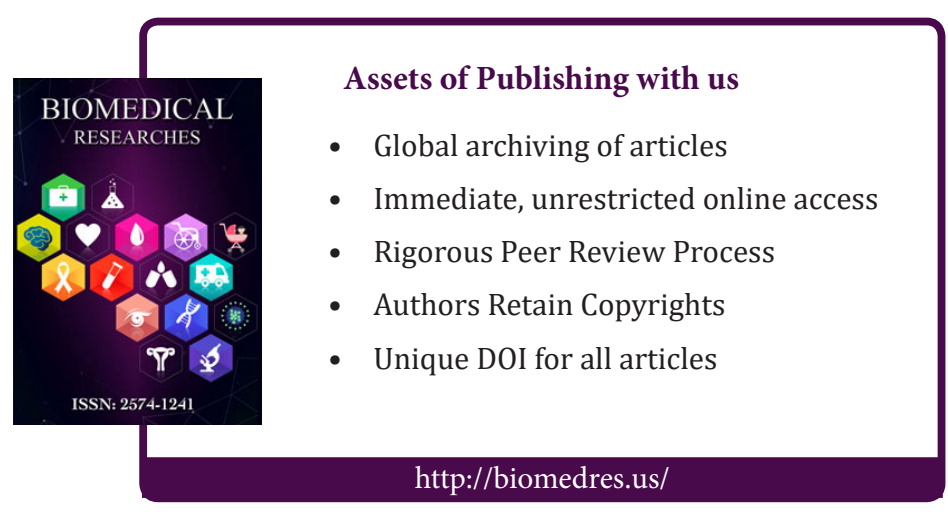

